\title{
On the accuracy of emitter localization method based on multipath exploitation in realistic scenarios
}

Md Abdullah Al Imran

Eray Arik

Yaser Dalveren ( $\nabla$ yaser.dalveren@atilim.edu.tr )

Mehmet Baris Tabakcioglu

Ali Kara

\section{Research Article}

Keywords: digital terrain elevation data, geographic information system, localization, multipath, radar, raytracing

Posted Date: December 8th, 2021

DOI: https://doi.org/10.21203/rs.3.rs-1140082/v1

License: (c) This work is licensed under a Creative Commons Attribution 4.0 International License.

Read Full License

Version of Record: A version of this preprint was published at Journal of Electromagnetic Waves and Applications on May 4th, 2022. See the published version at https://doi.org/10.1080/09205071.2022.2070031. 
On the accuracy of emitter localization method based on multipath exploitation in realistic scenarios

M. A. Al Imran ${ }^{\mathrm{a}}$, E. Arık ${ }^{\mathrm{b}}$, Y. Dalveren ${ }^{\mathrm{c} *}$, M. B. Tabakcioglu ${ }^{\mathrm{b}}$, and A. Kara $^{\mathrm{d}}$

${ }^{a}$ Department of Electrical and Electronics Engineering, Atilim University, Ankara, Turkey; ${ }^{b}$ Department of Electrical and Electronics Engineering, Bursa, Turkey;

${ }^{c^{*} D e p a r t m e n t}$ of Avionics, Atilim University, Ankara, Turkey; ${ }^{d}$ Department of Electrical and Electronics Engineering, Gazi University, Ankara, Turkey

*corresponding author: Yaser Dalveren (yaser.dalveren@atilim.edu.tr) 


\title{
On the accuracy of emitter localization method based on multipath exploitation in realistic scenarios
}

\author{
This study aims to evaluate the accuracy of a method proposed for passive \\ localization of radar emitters around irregular terrains with a single receiver in \\ Electronic Support Measures (ESM) systems. Previously, only the theoretical \\ development of the localization method was targeted by the authors. In fact, this \\ could be a serious concern in practice since there is no evidence about its \\ accuracy under the real data gathered from realistic scenarios. Therefore, firstly, \\ an accurate ray-tracing algorithm is adapted to the method in order to enable its \\ implementation in practice. Then, scenarios are determined based on the \\ geographic information system (GIS) map generated to collect high resolution \\ digital terrain elevation data (DTED) as well as realistic localization problems for \\ radar emitters. Next, the improved method is tested with simulations, and thus, its \\ performance is verified for practical implementation in Electronic Warfare (EW) \\ context for the first time in the literature. Lastly, based on the simulation results, \\ the performance bounds of the method are also discussed.
}

Keywords: digital terrain elevation data; geographic information system; localization; multipath; radar; ray-tracing.

\section{Introduction}

Determining the position of the source of emissions (emitters) is a proliferating demand for both military and civilian applications. It is widely known that the position of an emitter can be determined by employing well-known localization methods or algorithms which basically use sensor measurements and different sources of information [1]. Conventional localization methods such as angle of arrival (AOA), time difference of arrival (TDOA), and frequency difference of arrival (FDOA) utilize the received signals propagated through the direct paths from the source or emitter. In AOA-based localization, the bearing measurements from the radiated signal at a number of distributed sensors are used in order to estimate the emitter position [2]. In TDOAbased localization, the time differences of radiated signal from a stationary emitter to 
the number of distributed sensors are measured. Obviously, each time difference defines a hyperbola where the emitter could be located. The position of the emitter is then estimated by taking the intersection of the hyperbolas [3]. In order to estimate the position of the moving emitters, FDOA-based localization methods are developed. The FDOA method can be applied together with the TDOA, resulting in estimation of both the position and velocity of the emitter [4]. Moreover, the AOA method can also be combined with the TDOA method for localization [5]. However, in practice, multipath scattering is one of the most important concerns and it adversely affects the performance of those localization methods. For this reason, several multipath mitigation techniques have been proposed so far to suppress the multipath scattering effects [6$11]$.

Recently, on the other hand, researchers have intended to find out a way of exploiting multipath in emitter localization. These studies can be grouped into two main categories: (a) multiple sensor (receiver) localization system [12-15], (b) single receiver localization system [15-23]. Today, multipath-assisted localization of emitters by using the single receiver localization system remains an interesting subject of investigation. The methods developed within this context enable low-cost deployment, and provide accurate solutions. These advantages encourage initiatives to seek opportunities for development of different localization methods to overcome challenging operational situations.

\subsection{Related Works}

One of the existing studies in the literature is proposed in [15] which address a localization problem in the presence of multipath where the emitted signal is exposed to reflection from a surface. The location of emitting source(s) is estimated by using a particle filtering algorithm which processes AOA and TDOA measurements. In [16], a 
single receiver (base station) and the signal reflections from the building walls are used to localize and track a moving agent. It is verified that accurate localization in indoor environment can be achieved by using tracking algorithms both with and without data association. The work proposed in [17] derives a time of arrival (TOA) based algorithm in order to associate multipath reflections to their respective walls in urban sensing and through-the-wall (TTW) radar localization. A nonlinear least squares (NLS) approach is also derived to reduce the propagation effects for accurate localization. The developed algorithm assumes a condition that is the resolvability of all bounce multipath components. In order to relax this requirement, the work in [19] proposes a localization approach that uses the embedded directivity in ultra-wideband (UWB) antennas for estimating the target position. More specifically, the given approach uses pulse shape dependency on direction of arrival (DOA) of the radar return. Hence, it is shown that the availability of one single-bounce component along with the radar return is sufficient condition for localization. In [18], a method to localize a source via multipath exploitation in a known environment such as urban settings is proposed. It is presented that source localization with a known waveform in a three-dimensional volume can be achieved when there are minimum five TOAs. Another work is presented by Giacometti et al. which aims to exploit AOA and TDOA measurements for radar emitter localization problem in a naval context given that there is no any prior knowledge on the location of the reflectors [20]. In order to solve this problem, it proposes a method that is based on an assignment algorithm. In [21], a localization method is developed by considering a dominant object as an electromagnetic scatterer. In this method, the received direct and scattered waves through two different antenna systems are analyzed to estimate the location of the target. The study presented in [22] proposes a method that combines the channel impulse response (CIR) estimation and ray-tracing (RT) 
simulation tool. In the proposed method, the positions of the scatterers are mapped by using RT simulation which provides the information related to the ray paths. This information along with the time delay estimation is then used to determine the location of an emitter in non-line-of-sight (NLoS) environments. As a summary, the relevant works are listed in Table 1. As can be deduced from Table 1, most of the works employ only specular reflection for exploiting multipath. Besides, these works are mostly proposed to be used in indoor or urban applications.

Table 1. Summary of the relevant works on the use of multipath in emitter localization by single receiver.

\begin{tabular}{|c|c|c|c|c|}
\hline Reference & $\begin{array}{c}\text { Signal } \\
\text { Information } \\
\end{array}$ & $\begin{array}{l}\text { Environment/ } \\
\text { Application }\end{array}$ & Mechanism & Localization Approach \\
\hline$[15]$ & TDOA\&AOA & $\begin{array}{c}\text { Multipath } \\
\text { environments }\end{array}$ & Reflection & Particle filtering algorithm \\
\hline$[16]$ & TOA & $\begin{array}{c}\text { Office } \\
\text { environment }\end{array}$ & $\begin{array}{l}\text { Specular } \\
\text { reflection } \\
\text { from the } \\
\text { walls }\end{array}$ & $\begin{array}{c}\text { Tracking algorithm both with } \\
\text { and without } \\
\text { data association }\end{array}$ \\
\hline [17] & TOA & $\begin{array}{l}\text { Urban sensing } \\
\text { and TTW } \\
\text { radar }\end{array}$ & $\begin{array}{l}\text { Specular } \\
\text { reflection } \\
\text { from the } \\
\text { walls }\end{array}$ & $\begin{array}{l}\text { TOA wall assosication } \\
\text { algorithm \& NLS }\end{array}$ \\
\hline [19] & TOA\&DOA & $\begin{array}{l}\text { Urban sensing } \\
\text { and TTW } \\
\text { radar }\end{array}$ & $\begin{array}{l}\text { Specular } \\
\text { reflection } \\
\text { from the } \\
\text { walls }\end{array}$ & $\begin{array}{l}\text { A method using pulse shape } \\
\text { dependency on DOA } \\
\text { of the radar return }\end{array}$ \\
\hline$[18]$ & TOA & Urban settings & $\begin{array}{l}\text { Specular } \\
\text { reflection } \\
\text { from the } \\
\text { walls }\end{array}$ & $\begin{array}{l}\text { The Bancroft method-based } \\
\text { algorithm }\end{array}$ \\
\hline$[20]$ & TDOA\&AOA & Naval context & $\begin{array}{l}\text { Specular } \\
\text { reflection } \\
\text { from point } \\
\text { reflectors }\end{array}$ & Assignment algorithm \\
\hline$[21]$ & TDOA\&DOA & $\begin{array}{l}\text { Scenarios with } \\
\text { a large well- } \\
\text { known object }\end{array}$ & $\begin{array}{c}\text { Scattering } \\
\text { from a } \\
\text { cylinder }\end{array}$ & $\begin{array}{l}\text { Electromagnetic } \\
\text { modelling/analysis }\end{array}$ \\
\hline$[22]$ & TDOA & $\begin{array}{l}\text { NLoS } \\
\text { environments }\end{array}$ & $\begin{array}{l}\text { Diffraction } \\
\text { and specular } \\
\text { reflection } \\
\text { from the } \\
\text { walls }\end{array}$ & $\begin{array}{c}\text { Combination of RT } \\
\text { simulation tool and the CIR } \\
\text { estimation }\end{array}$ \\
\hline$[23]$ & TOA\&AOA & ESM systems & $\begin{array}{c}\text { Diffuse } \\
\text { scattering } \\
\text { from } \\
\text { irregular } \\
\text { terrains } \\
\end{array}$ & $\begin{array}{c}\text { Weighted averaging method } \\
\text { based on both a GIS-assisted } \\
\text { RT algorithm and a TDOA } \\
\text { technique }\end{array}$ \\
\hline
\end{tabular}


On the other hand, only the work presented in [23] considers an advance localization system to be used in electronic warfare (EW) context. In the work, a novel approach is proposed for passive localization of radar emitters around irregular terrains in Electronic Support Measures (ESM) systems. Unlike the previous works, the approach involves diffuse scattering over irregular terrain in multipath exploitation. In the approach, the scatter regions over the irregular terrain where the multipath signals are scattered are used as virtual sensors. Moreover, the likelihoods of the scatter regions being a source of multipath signals are utilized through a ray-tracing algorithm along with the Geographic Information System (GIS). Therefore, a weighted averaging method based on the use of both a Ray-tracing algorithm and a TDOA technique are employed in order to determine the location of the emitter. Here, it is evident that the identification of the likelihoods of the multipath scattered signals is indicative for the localization accuracy. Thus, it can be inferred that it is necessary to utilize an accurate ray tracing algorithm to calculate the likelihoods. However, the work does not provide any ray-tracing algorithm due to the fact that it only aims at the theoretical development of the localization method. In this context, there might be serious concerns related to the performance of the proposed method under the real data gathered from realistic scenarios in practice. Hence, the proposed method needs not only to be enhanced by adapting a ray tracing algorithm, but also to be evaluated in terms of accuracy and performance under various realistic scenarios.

\subsection{Overview and Contributions}

This study is devoted to alleviate the concerns related to the performance of the localization method proposed in [23] under realistic scenarios. To this end, initially, a ray-tracing algorithm is adapted by using realistic obstruction models. To do this, some scenarios are constructed in some parts of the Aegean Sea. From each scenario, 
GIS map is generated to collect (Digital Terrain Elevation Data) DTED data which are then used in the ray tracing algorithm in order to calculate the likelihoods of the scatter regions being a source of multipath signals. Besides, the multipath scattering centers are determined and assumed as virtual sensors to be used in an efficient TDOA-based localization method, so-called improved two-step weighted least-squares (TSWLS) [24]. In order to account all virtual sensors along with the likelihoods for localization, a weighted averaging method is applied. Next, simulations in constructed realistic scenarios are conducted to study the applicability and validity of the proposed method. Thus, the performance bounds of the method in realistic scenarios are also scrutinized. Overall, the major contributions of the work can be listed as follows:

(1) A localization method based on improved-TSWLS is fully developed by adapting an accurate ray tracing algorithm.

(2) Scenarios are developed based on high resolution real terrain data (GIS) as well as realistic localization problems for radar emitters.

(3) The method is tested with simulations and its performance is verified for practical implementation in EW context.

\section{Basics of the localization method}

The problem considered in this article is related to the operational challenges of Electronic Support Measures (ESM) systems while locating radar emitter's position over and around the sea surface. One of the important challenges within this context is owing to the multipath scattering caused by irregular terrains such as islands and littorals. Specifically, while discriminating the radar emitter, multipath signals that have similar radar parameters in the radar field of view cause difficulties for the ESM system. This, then, constitutes a serious problem that adversely affects the localization 
performance. In order to gain a concrete understanding of the problem, Figure 1, shows a simplified two-dimensional (2D) problem space (top-view) where the altitudes are ignored.

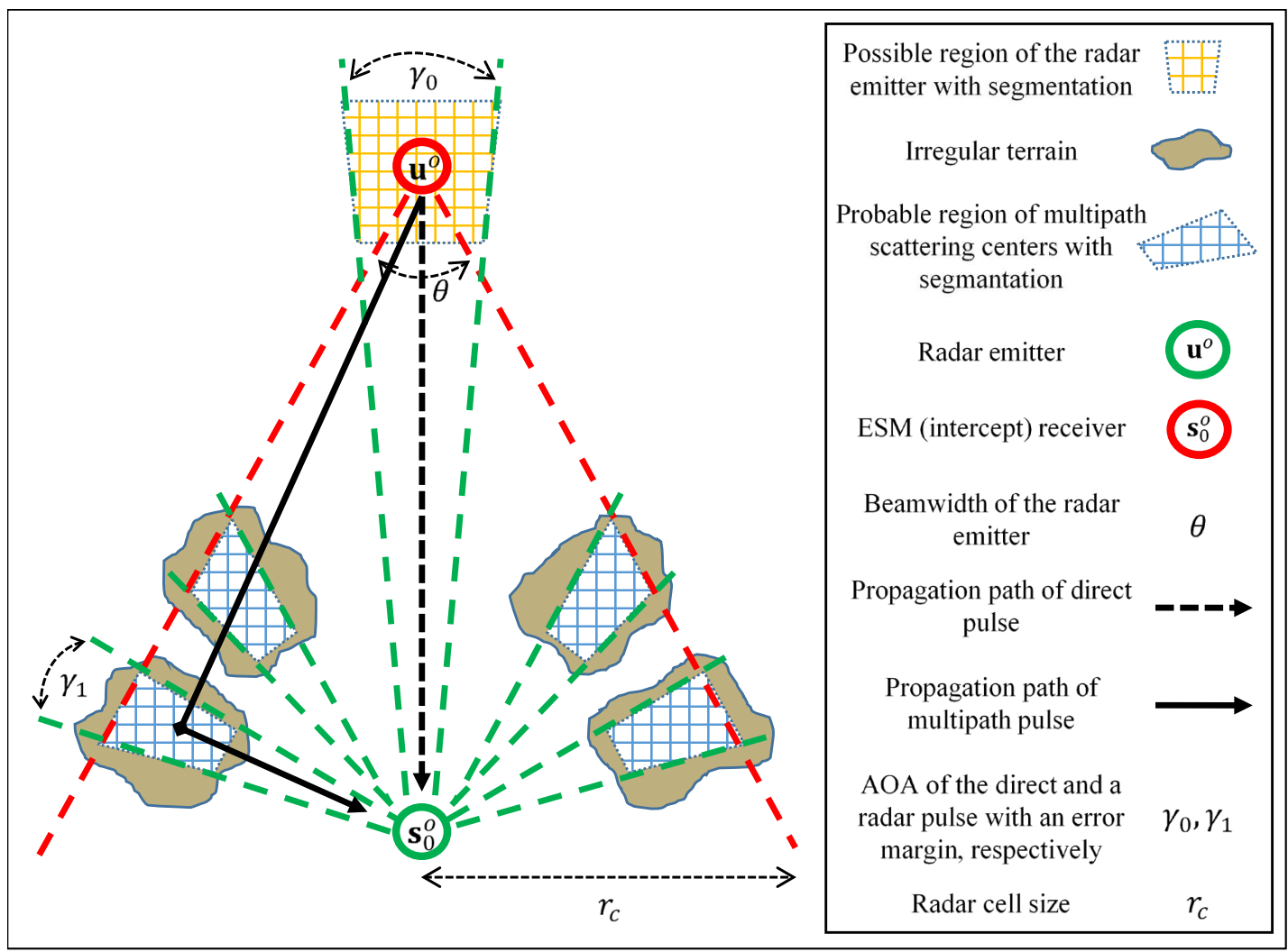

Figure 1. Problem space in 2D.

In Figure $1, \mathbf{u}^{o}=\left[x^{o}, y^{o}, z^{o}\right]^{T}$ and $\mathbf{s}_{0}^{o}=\left[x_{0}^{o}, y_{0}^{o}, z_{0}^{o}\right]^{T}$ denote the position of the radar transmitter and ESM (intercept) receiver. The irregular terrains are depicted by irregular circles with brown-color. For the sake of simplicity, only two propagation paths consisted of direct path $\left(t_{0}\right)$ and multipath $\left(t_{1}\right)$ are considered. Here, in order to solve the problem of emitter location from a single receiver using direct and multipath pulses, a novel method is proposed to use multipath effects as an advantage in localization of emitters [23]. Basically, the proposed method is based on an idea that aims to use multipath scattering centers as virtual sensors for determining emitter location. For this purpose, it employs an algorithm of which the main stages are briefly described in below. The flowchart of the algorithm is shown in Figure 2. 


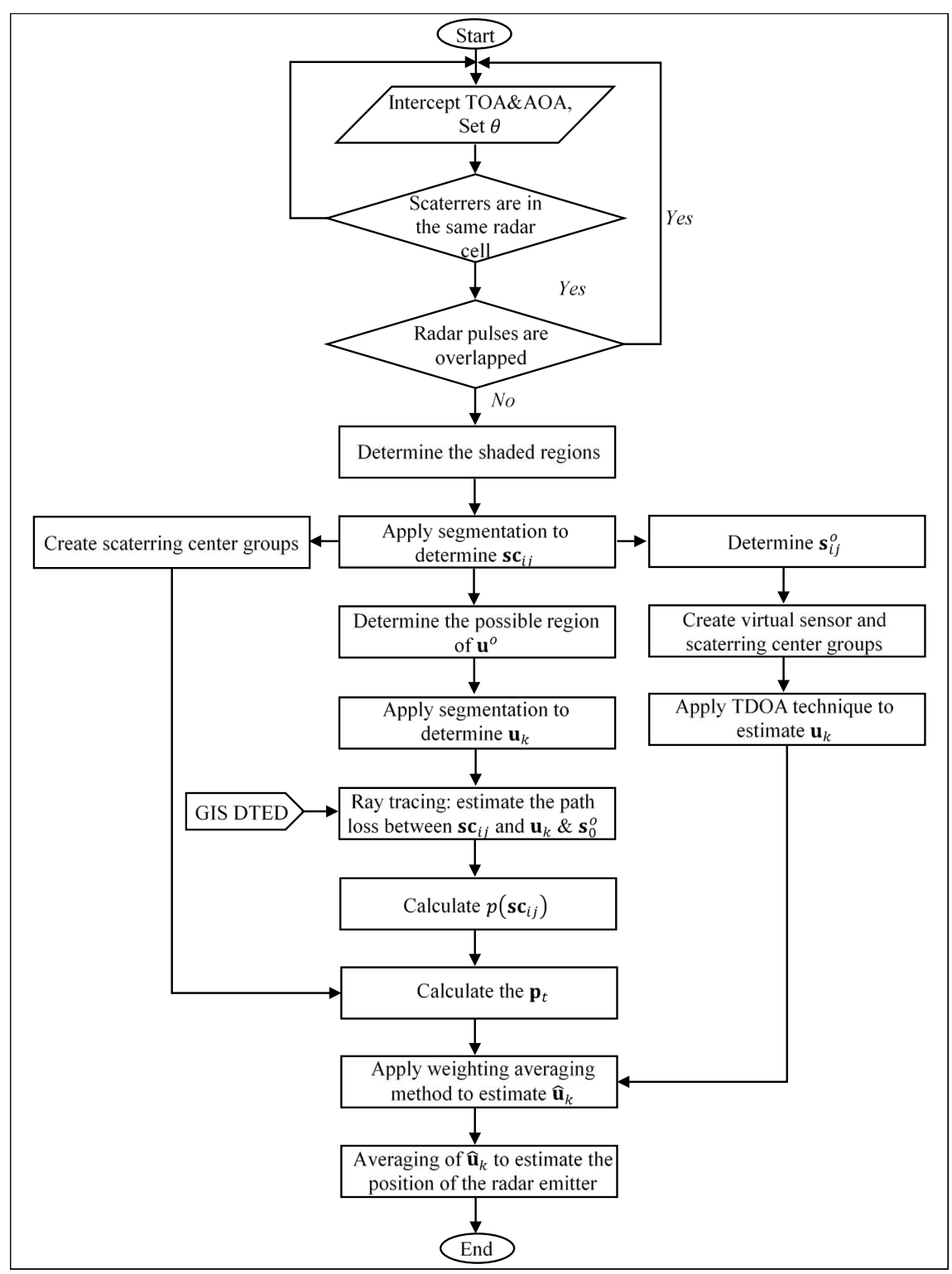

Figure 2. Flowchart of the localization algorithm.

\subsection{Stage I}

In this stage, multipath scattering centers are estimated. To this end, ESM receiver's AOA and TOA information are required for direct and multipath pulses. Hence, it is necessary to estimate the probable region of multipath scattering centers.

In the first step of this stage, the outer boundaries of the probable region of multipath scattering centers are determined. It is important to note that the scatterers should be determined in the same radar cell which is the most important requirement 
that should be satisfied. Here, the radar cell size $\left(r_{c}\right)$ corresponds to the projection on the ground since the $2 \mathrm{D}$ problem is considered.

In the second step, the inner boundaries of the probable region of multipath scattering centers are determined. This is achieved by satisfying another requirement that the pulses received from direct path and multipath should not be overlapped in time scale. In order to avoid such an overlapping, the difference of TOA measurements (or TDOA) between directly received and multipath pulses must be greater than the pulse width of the radar emitter. Therefore, it becomes possible to determine a minimum distance from the receiver to the inner boundary of the probable region of virtual sensors. Yet, notice that this distance can be determined only if the TDOA measurements are expressed in terms of range differences.

In the third stage, the width of the probable region of multipath scattering centers is specified by the AOA of radar pulses with an error margin $(\gamma)$ as shown in Figure 1. By incorporating the information achieved in all those steps, the probable region of multipath scattering centers over the rough surface are easily determined.

On the other hand, since the probable region of multipath scattering centers are considered as irregular terrains in practice, it is expected that the multipath pulses are exposed to diffuse scattering. Hence, the local region from which the multipath pulses are scattered could be then a multipath scattering center. Thus, in the last step, segmentation is applied as shown in Figure 1 to split up the probable region of multipath scattering centers so that each of segments can be considered as multipath scattering centers $\left(\mathbf{s c}_{i j}, i=1, \ldots, N_{j}\right.$ and $j=1, \ldots, M$ where $N_{j}$ denotes the number of the scattering centers in the $j^{\text {th }}$ probable region of virtual sensors, and $M$ denotes the number of probable region of virtual sensors). Among these, however, further process is required to determine the proper ones that can be utilized in the localization. This, in 
fact, can be achieved by calculating the likelihood of the segments from which the multipath scattering takes place as discussed in the next stage.

\subsection{Stage II}

In this stage, it is aimed to calculate the likelihood of the scattering centers being a source of the multipath scattering $p\left(\mathbf{s c}_{i j}\right)$ by utilizing a ray tracing algorithm. It is important to note that the work presented in [23] lacks of providing a ray-tracing algorithm. It only proposes the conceptual structure of the algorithm. Therefore, this study attempts to fulfill this gap by providing an accurate ray tracing algorithm. In the following, theoretical background of the algorithm is presented, and then, the adaptation of the algorithm to the localization problem is described which is followed by the calculation of the likelihood of the scattering centers.

\subsubsection{Background information}

Computational electromagnetics for high frequency can be categorized into field- and current-based groups [25]. The latter employs Physical Optics (PO) for shortwavelength approximation. Field-based methods, on the other hand, rely on Geometric Optics (GO) and well suited for the typical radar frequencies of the problem space and utilize ray tracing methods [26]. The ray tracing methods can be divided into two main categories, namely image method and ray launching method. The image method based on symmetry with respect to segments. The image method controls whether the rays are blocked by the segments or not. If the number of the segments is higher so that problem will be harder and requires so much time and CPU usage. The authors have already studied various aspects of ray tracing algorithms, and extensively published [27-29]. Hence, using the ray launching method could be a good option instead of image method for wireless propagation prediction when the localization problem is considered. 
Therefore, in this study, ray launching method is used due to its advantageous over the image method.

Basically, the ray launching method can be applied in three steps: (1) ray initiation, (2) ray tracing and (3) ray interception [30]. In the ray initiation step, the rays are launched with the same differential angle intervals. In the ray tracing step, the rays are controlled to determine whether the rays are blocked by an obstacle or not. If the rays are blocked by an obstacle, they are reflected by this obstacle with the same angle of incidence. Here, the number of reflection is determined by the user. If there is no reflection for the first ray from the obstacles, then it is passed to the second ray. After all the rays are traced in this step, the rays are intercepted in a receiver position.

In practice, on the other hand, the direct, reflected or diffracted wave can be measurable at the receiver. In this case, electric field of the direct wave can be expressed as [31]

$$
E_{\text {dir }}=\frac{1}{r_{0}} e^{-j k r_{0}}
$$

where $r_{0}$ is distance between the transmitter and receiver, and $k$ is the wave number. Besides, electric field of the reflected waves can be expressed as

$$
E_{r e f}=R \frac{1}{r} e^{-j k r}
$$

where $r$ is the total distance of the transmitter, ground and the receiving point, and $R$ is the reflection coefficient. Moreover, according to the Uniform theory of diffraction (UTD) model, electric field of the diffracted waves can be expressed as [32]

$$
E_{\text {dif }}=E_{\text {in }} D A(r) e^{-j k r},
$$

where $E_{\text {in }}$ is incoming field, $A$ is the spreading factor, and $D$ is the diffraction coefficient which can be expressed as [33] 


$$
D(\alpha)=-\frac{e^{-j \pi / 4}}{2 \sqrt{2 \pi \cos (\alpha / 2)}} F[x]
$$

where $\alpha$ is the diffraction angle and $F[x]$ is the transition function given as [34]

$$
F[x]=2 j \sqrt{x} e^{-j x} \int_{\sqrt{x}}^{\infty} e^{-j u^{2}} d u,
$$

where $x=2 k L \cos ^{2}(\alpha / 2)$, and $L$ is the distance parameter given as

$$
L=\frac{r_{0} r_{1}}{r_{0}+r_{1}}
$$

\subsubsection{Implementation of the ray tracing algorithm}

It is strictly necessary that the scattering centers are electromagnetically visible to both the radar emitter and the receiver in order to use a ray tracing algorithm in the proposed localization method. In this context, the probable region of the radar emitter needs to be defined properly. To do this, the distance between the radar emitter and the receiver is estimated by utilizing both the peak transmitting power of the radar emitter and the received power as well as the erroneous AOA of the radar pulses measured by the receiver $\left(\gamma_{i}\right)$. Because of imperfections that presence in the radar emitter or the receiver, the estimated distance is expected to be erroneous which corresponds to the width of the region in perpendicular to the direction to the receiver. Next, as shown in Figure 1, the region needs to be split up to segments so that the center of each segment can be assumed as the nominal position of the radar emitter, $\mathbf{u}_{k}=\left[x_{k}, y_{k}, z_{k}\right], k=1, \ldots, K$ where $K$ is the number of segments of the probable region of the radar emitter. This may then provide an opportunity to construct the links between the scattering center and the nominal position of the radar emitter. To do that, the center of each scattering center is needed to be determined, $\mathbf{s}_{i j}^{o}=\left[x_{i j}^{o}, y_{i j}^{o}, z_{i j}^{o}\right]$. In this way, the links between the scattering center and the receiver can also be constructed. After constructing the links, it 
becomes possible to implement the ray tracing algorithm to estimate how the obstacles might cause path loss for the particular links.

In order to implement the ray tracing algorithm, firstly, the area where the localization method is expected to employ is determined in a 3D map. Here, the algorithm requires utilizing DTED which can be achieved by GIS. The DTED is a raster topographic data that provides basic quantitative data for the systems requiring terrain elevation, slope, and surface information. Here, selecting the higher level of DTED tiling scheme is crucial to gather precise terrain data. Then, for constructing the links between the nominal positions of the radar emitter and the center of each scattering center, and for the links between the center of each scattering center and the receiver, a 2D terrain profile is extracted from 3D map, and it is redefined with line segmentation to apply the ray tracing algorithm. Figure 3 shows a typical 2D terrain profile and line segmentation.

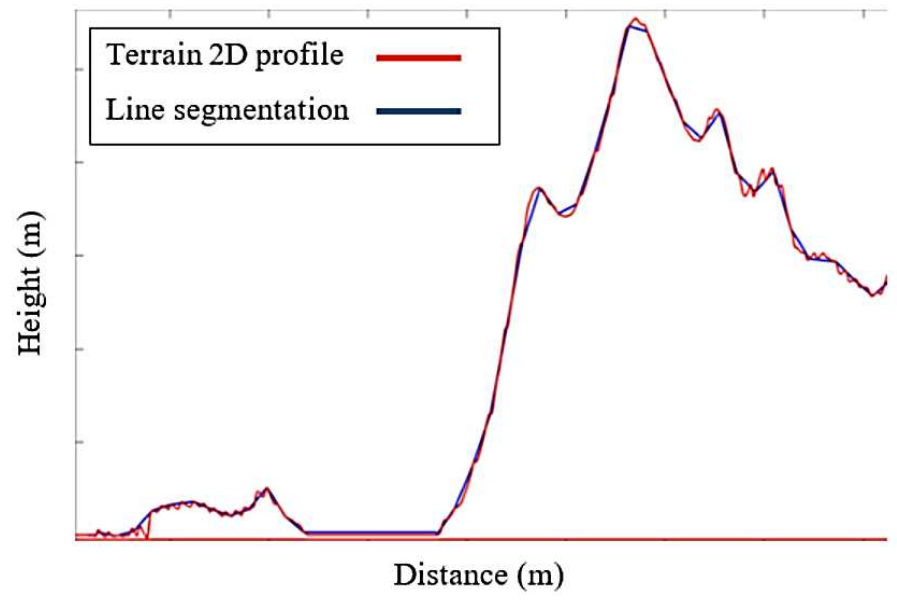

Figure 3. A 2D profile and line segmentation.

Next, for the links between the nominal positions of the radar emitter and the center of each scattering center, the transmitting and receiving points are deployed at the nominal positions of the radar emitter and the center of each scattering center, respectively. Similarly, for the links between the center of each scattering center and the receiver, the transmitting and receiving points are deployed at the center of each 
scattering center and the receiver, respectively. In both links, all the direct, the reflected and the diffracted waves are determined by using the ray tracing algorithm. Thus, the electric field and so coverage map for a 2D profile is calculated with using all the direct, reflected and diffracted ray contributions. The path loss for each link can be then estimated. As an example, a coverage map for a 2D terrain profile is illustrated in Figure 4.

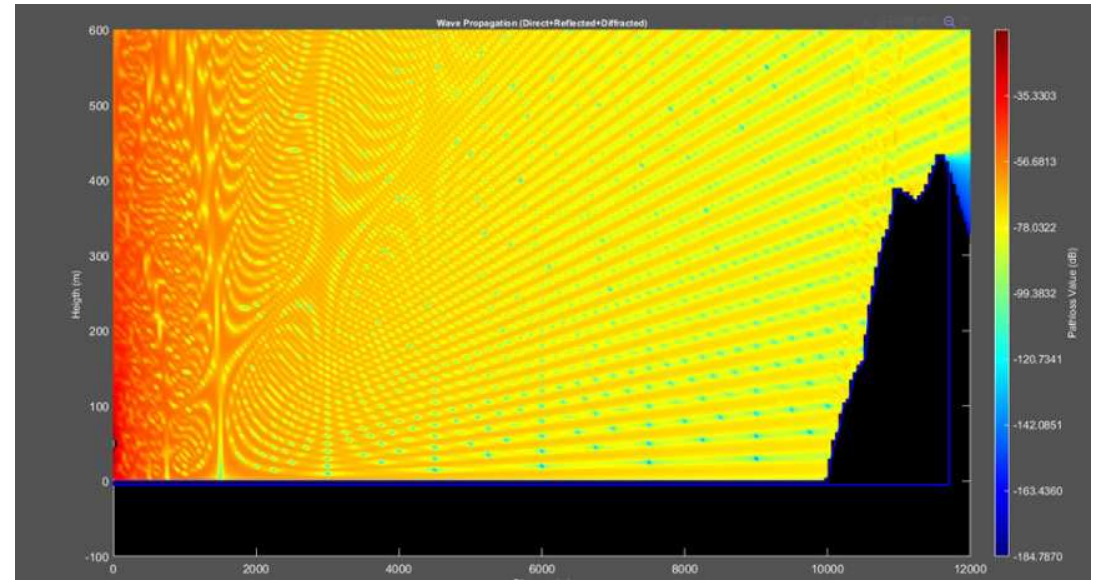

Figure 4. A sample coverage map for 2D terrain profile.

It is seen in Figure 4, coverage map is obtained by using all the direct, diffracted and reflected waves. Moreover, path loss is reduced with far away from the transmitter. Furthermore, behind the obstacle, there are no reflected or direct waves. In this case, there are only diffracted waves. Electric field of the diffracted wave is reduced sharply as it is illustrated. It is worth noting that the obstructions are expected to fall inside the Fresnel zone generated between the nominal position of the radar emitter and the receiver. Moreover, because of the considerations regarding to determination of the probable region of multipath scattering centers discussed in Stage I, the obstructions are also expected to fall inside the vertical plane containing the nominal position of the radar emitter and the receiver. It is also important to note that the reflecting surface on the obstacle is assumed to be locally flat (line segment). In this case, specular reflection also known as the Fresnel approximation is expected to be obtained. Besides, the 
diffraction is also expected to be obtained owing to the presence of edge or wedgeshaped obstacles between the transmitter and the receiver. Therefore, both the reflected and diffracted waves from the obstructions are measurable at the receiver.

\subsubsection{Calculating the likelihood of the scattering centers}

Calculating the likelihood of the scattering centers, $p\left(\mathbf{s c}_{i j}\right)$, has a key role in the implementation of the localization method. In practice, there may be different propagation mechanisms for each of segments of the probable region of virtual sensors. For this reason, total path loss (or path gain) should be accounted to calculate the likelihoods. For the considered problem, the path loss for a particular link is consisted of the free-space path loss (FSPL), and also the reduction in the received electric field due to reflection $\left(P L_{r}\right)$ and the loss due to diffraction $\left(P L_{d}\right)$ which are provided by the ray-tracing algorithm. If there is no obstruction between the transmitter and the receiver position, FSPL is used to calculate the field strength as in Eq.(1). Moreover, electric field of single or multiple reflected wave from the line segments can be calculated $P L_{r}$ as in Eq.(2). Furthermore, from the sharp surfaces, there is diffraction, and field strength of the diffracted waves is obtained by the Eq.(3). Basically, the path loss $(P L)$ for a fundamental link can be then expressed as

$$
P L[\mathrm{~dB}]=F S P L+P L_{r}+P L_{d}
$$

As can be expected, there may be multiple reflected or diffracted waves reaching to the receiver. The developed ray tracing algorithm determines multiple reflection $(\mathrm{R})$ and diffraction (D) rays up to 6 times (for example, reflection-diffraction-reflectionreflection-diffraction-diffraction) though the radar emitter localization problem requires only a few. 
It is important to note that the factors for calculating the $P L$ is can be varied according to the localization scenario. In some cases, the diffracted signals become unmeasurable due to the position of the obstacles. Then, the $P L$ simply consists of only the FSPL and $P L_{R}$.

Evidently, the $P L$ needs to be estimated for all the scattering centers in each probable region of virtual sensors. From a general perspective, the path gain $(P G)$ value of the $i^{\text {th }}$ scattering center in the $j^{\text {th }}$ probable region of scattering centers $\left(P L_{i j}\right)$ can be estimated by

$$
P G_{i j}=1 / P L_{i j}
$$

where

$$
P L_{i j}=P L_{\mathbf{u}_{k} \mathbf{s c}_{i j}}+P L_{\mathbf{s} \mathbf{c}_{i j} \mathbf{s}_{0}^{o}}
$$

where $P L_{i j}$ is the total path loss of the link between the nominal position of the radar emitter and the center of each scattering center $\left(P L_{\mathbf{u}_{k} \mathbf{s} \mathbf{c}_{i j}}\right)$, and the link between the center of each scattering center and the receiver $\left(P L_{\mathbf{u}_{k}} \mathbf{s c}_{i j}\right)$.

Therefore, the estimated losses can be used to calculate the $p\left(\mathbf{s c}_{i j}\right)$ as follows

$$
p\left(\mathbf{s c}_{i j}\right)=P G_{i j} / \sum_{i=1}^{N_{j}} P G_{i j}
$$

\subsection{Stage III}

In the third stage, the purpose is to estimate the position of the radar emitter. The steps required to achieve this goal is straightforward. In the first step, the center of each scattering center determined in Stage I are considered as the nominal positions of the virtual sensors. The single-sensor localization problem then becomes a multiple-sensor localization problem which can be solved by conventional techniques using TDOA measurements. However, as the multipath scattering involves diffuse components over 
the irregular terrain, an uncertainty is expected in the location of the scattering centers. In this case, the erroneous virtual sensor positions are needed to take into account. For this reason, it is necessary to employ an efficient source localization technique using TDOA measurements in the presence of sensor position errors. From the comparison results provided in [35], the improved two-step weighted least-squares (improvedTSWLS) technique presented in [24] could be a good choice to be utilized in the proposed localization method due to its efficiency at high sensor position errors. In the following section, adaptation of improved-TSWLS technique to the proposed method is briefly described. This is followed by another section to discuss the second step of this stage which presents the weighting averaging method used for final estimate of the radar emitter.

\subsubsection{Adaptation of improved-TSWLS technique}

In order to comprehend the adaptation of the improved-TSWLS technique to the proposed method, we should consider the problem space as illustrated in Figure 1. In the figure, assume that there is only one virtual sensor in each of probable regions of scattering centers and there is only one nominal position of the radar emitter $(k=1)$ for the sake of simplicity. In this case, by considering 3D, there are $M$ virtual sensors at locations $\mathbf{s}_{j}^{o}=\left[x_{j}^{o}, y_{j}^{o}, z_{j}^{o}\right]^{T}$ where $j=0, \ldots, M$, and the receiver, $\mathbf{s}_{0}^{o}$, in order to locate the radar emitter, $\mathbf{u}^{o}$. Here the case that $j=0$ can be considered as the position of the receiver while the other values correspond to the positions of virtual sensors. In order to account the uncertainty in the location of the scattering centers, the virtual sensor positions are needed to be considered as noisy. Therefore, sensor position vector can be defined as $\mathbf{s}=\left[\mathbf{s}_{0}^{T}, \mathbf{s}_{1}^{T}, \ldots, \mathbf{s}_{M}^{T}\right]^{T}=\mathbf{s}^{o}+\Delta \mathbf{s}$ where $\mathbf{s}^{o}=\left[\mathbf{s}_{0}^{o^{T}}, \mathbf{s}_{1}^{o^{T}}, \ldots, \mathbf{s}_{M}^{o^{T}}\right]$ and $\Delta \mathbf{s}$ is the sensor position error vector, $\Delta \mathbf{s}=\left[\Delta \mathbf{s}_{0}{ }^{T}, \Delta \mathbf{s}_{1}{ }^{T}, \ldots, \Delta \mathbf{s}_{M}{ }^{T}\right]^{T}$, which is assumed to be a 
zero-mean random variable and Gaussian distributed with the covariance matrix, $\mathbf{Q}_{s}=$ $E\left[\Delta \mathbf{s} \Delta \mathbf{s}^{T}\right]$. Since the position of the $\mathbf{s}_{0}^{o}$ is exactly known, the value of $\Delta \mathbf{s}_{0}$ should be defined as approximately zero.

In order to employ the improved-TSWLS technique, the $\mathbf{s}_{0}^{o}$ is assigned as the reference sensor. In this case, the TDOAs is expressed as

$$
\Delta t_{j+1,0}=\left(t_{j+1}-t_{0}\right)
$$

where $t_{0}$ and $t_{j+1}$ denote the time of arrival to the reference sensor and the $j^{\text {th }}$ sensor, respectively. Then, the TDOAs can be converted to the range differences (RDs) by

$$
r_{j+1,0}^{o}=c \Delta t_{j+1,0}=c\left(t_{j+1}-t_{0}\right)=r_{j+1}^{o}-r_{0}^{o}
$$

where $c$ is the velocity of light, $r_{j+1}^{o}$ denote the Euclidian distance between the emitter and the $j^{\text {th }}$ sensor, and $r_{0}^{o}$ is the Euclidian distance between the emitter and the $\mathbf{s}_{0}^{o}$.

Moreover, TDOA or RD measurement vector can be defined as $\mathbf{r}=$ $\left[r_{1,0}, r_{2,0}, \ldots, r_{M, 0}\right]^{T}=\mathbf{r}^{o}+\Delta \mathbf{r}$ where $\mathbf{r}^{o}=\left[r_{1,0}^{o}, r_{2,0}{ }^{o}, \ldots, r_{M, 0}{ }^{o}\right]^{T}$ and $\Delta \mathbf{r}$ is the measurement error vector, $\Delta \mathbf{r}=\left[\Delta r_{2,0}, \Delta r_{3,0}, \ldots, \Delta r_{M, 0}\right]$, which is assumed to be a zeromean random variable and Gaussian distributed with the covariance matrix, $\mathbf{Q}_{t}=$ $E\left[\Delta \mathbf{r} \Delta \mathbf{r}^{T}\right]$

In improved-TSWLS technique, basically, there are two stages in order to locate the emitter. In the first stage, by introducing nuisance parameters, a non-linear measurement equation is linearized and then solved. To this end, firstly an unknown vector, $\mu_{1}$, containing preliminary estimates of the radar emitter and the distance between the emitter and the receiver are defined. Then, the weighted least-squares estimate of vector $\mu_{1}$ is

$$
\mu_{1}=\left(\mathbf{G}_{1}^{T} \mathbf{W}_{1} \mathbf{G}_{1}\right)^{-1} \mathbf{G}_{1}^{T} \mathbf{W}_{1} \mathbf{h}_{1}
$$

where 


$$
\mathbf{G}_{1}=-2\left[\begin{array}{cc}
\left(\mathbf{s}_{1}-\mathbf{s}_{0}\right)^{T} & r_{1,0} \\
\vdots & \vdots \\
\left(\mathbf{s}_{M}-\mathbf{s}_{0}\right)^{T} & r_{M, 0}
\end{array}\right], \quad \mathbf{h}_{1}=\left[\begin{array}{c}
r_{1,0}^{2}-\mathbf{s}_{1}^{T} \mathbf{s}_{1}+\mathbf{s}_{0}^{T} \mathbf{s}_{0} \\
\vdots \\
r_{M, 0}^{2}-\mathbf{s}_{M}^{T} \mathbf{s}_{M}+\mathbf{s}_{0}^{T} \mathbf{s}_{0}
\end{array}\right]
$$

and

$$
\mathbf{W}_{1}=\left(\mathbf{B}_{1} \mathbf{Q}_{t} \mathbf{B}_{1}^{T}+\mathbf{D}_{1} \mathbf{Q}_{s} \mathbf{D}_{1}^{T}\right)^{-1}
$$

where

$$
\mathbf{B}_{1}=2 \operatorname{diag}\left[r_{1}^{o}, \ldots, r_{M}^{o}\right]
$$

where $\operatorname{diag}[\cdot]$ denotes the diagonal matrix.

In (13), $\mu_{1}(1: 3)$ denotes first three elements of $\mu_{1}$, and corresponds to preliminary estimate of the radar emitter. Additionally, $\mu_{1}(4)$ is the last element of $\mu_{1}$, which is the preliminary estimate of the distance between the emitter and the receiver.

In the second stage, the localization error of the first stage is estimated and then it is subtracted from the output of the first stage in order to provide the result. For this purpose, second stage begins with updating (13) as

$$
\mu_{2}=\left(\mathbf{G}_{2}^{T} \mathbf{W}_{2} \mathbf{G}_{2}\right)^{-1} \mathbf{G}_{2}^{T} \mathbf{W}_{2} \mathbf{h}_{2}
$$

where

$\mathbf{G}_{2}=\left[\begin{array}{c}\mathbf{I}_{(3,3)} \\ -\left(\left(\mu_{1}(1: 3)-\mathbf{s}_{0}\right) /\left\|\left(\mu_{1}(1: 3)-\mathbf{s}_{0}\right)\right\|\right)\end{array}\right], \mathbf{h}_{2}=\left[\begin{array}{c}\mathbf{0}_{(3,1)} \\ \mu_{1}(4)-\left\|\left(\mu_{1}(1: 3)-\mathbf{s}_{0}\right)\right\|\end{array}\right]$,

and

$$
\mathbf{W}_{2}=\mathbf{B}_{2}\left(\mathbf{G}_{1}^{T} \mathbf{W}_{1} \mathbf{G}_{1}\right)^{-1} \mathbf{B}_{2}^{T}
$$

where

$$
\mathbf{B}_{2}=\left[\begin{array}{cc}
-\mathbf{I}_{(3,3)} & \mathbf{0}_{(3,1)} \\
\mathbf{0}_{(3,1)}^{T} & 1
\end{array}\right]
$$

Here, $\mathbf{I}_{(3,3)}$ is $3 \times 3$ identity matrix while $\mathbf{0}_{(3 \times 1)}$ is $3 \times 1$ zero vector. Hence, the emitter location is estimated as

$$
\widehat{\mathbf{u}}=\mu_{1}(1: 3)-\mu_{2} .
$$




\subsubsection{Weighting averaging method}

Obviously, in practice, there are several number of virtual sensors in each of probable regions of scattering centers, and there are several number of nominal position of the radar emitter in the probable region of the radar emitter. Then, the localization problem becomes complicated and further approaches are required to solve the problem. For this purpose, as a first step, virtual sensor groups are created by considering all the combinations of virtual sensors selected from $M$ probable region of scattering centers in order to employ the improved-TSWLS technique. In this way, scattering center groups are also created. Thus, it becomes possible to calculate the likelihood of the scattering center groups $\left(\mathbf{p}_{t}, t=1, \ldots, T\right.$ where $T$ is the number of the combinations of scattering centers or virtual sensors) by considering the reliability of series system [36]. Then, for each of the virtual sensor groups, the nominal position of the radar emitter is estimated by employing improved-TSWLS technique

$$
\widehat{\mathbf{u}}_{k, t}=\left[\begin{array}{llll}
\widehat{\mathbf{u}}_{k, 1} & \widehat{\mathbf{u}}_{k, 2} & \cdots & \widehat{\mathbf{u}}_{k, T}
\end{array}\right] \quad \text { for } k=1, \ldots, K
$$

where $K$ is the number of segments of the probable region of the radar emitter.

Thus, in order to estimate the position of the emitter, the weighted averaging method is used as follows

$$
\overline{\mathbf{u}}_{k}=\sum_{t=1}^{T} \mathbf{p}_{t} \times \widehat{\mathbf{u}}_{k, t},
$$

and, the averaging of all estimated positions gives the final estimate of the emitter position $(\overline{\mathbf{u}})$

$$
\overline{\overline{\mathbf{u}}}=\left(\sum_{k=1}^{K} \overline{\mathbf{u}}_{k}\right) / K
$$

As a final note, although the proposed localization method is based on the use of TDOA method, it differs from the conventional localization methods which require 
multiple sensors (receivers), or, to be more precise, wireless sensor networks. It uses virtual sensors which are determined from the multipath scattering centers, and a single receiver that receives both direct and scattered signals to analyses them for estimating the position of the emitter. Thus, unlike conventional methods that need for communication links between the sensors (or between the emitter and sensors), it only requires to establish the links for the implementation of ray tracing algorithm as discussed earlier.

\section{Simulations}

This section presents the results of simulations conducted to examine the applicability and validity of the localization method under realistic scenarios. For creating the scenarios, a part of Aegean Sea is chosen from the 3D map as shown in Figure 5(a,b).

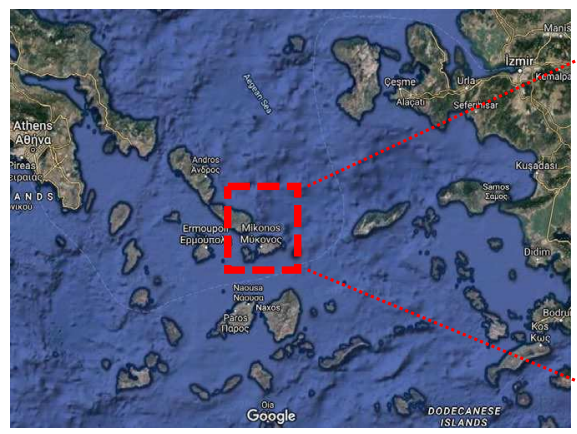

(a)

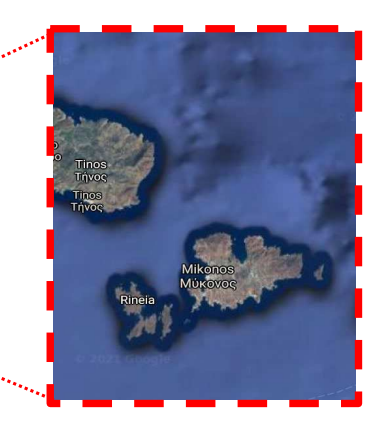

(b)

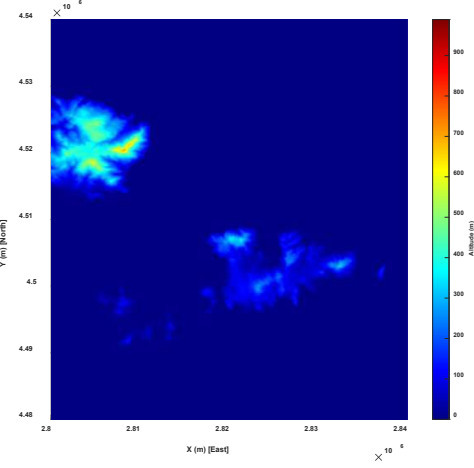

(c)

Figure 5. (a) Broad view of a part of Aegean Sea, (b) Region of interest, (c) GIS 3D map.

After determining a particular region over the Aegean Sea, GIS map was generated to use DTED data (Figure 5(c)). Three different simulation experiments containing various scenarios were then created by varying the receiver deployment around the scatterers. In the first experiment, the receiver was deployed behind of the scatterers while it was deployed at the center of the scatterers in the second experiment; and it was deployed at the front of the scatterers in the last experiment. As an example, 
Figure 6 shows the deployment of the receiver (rectangle) along with the emitter (triangle) and virtual sensors (circles) for a fixed radar beamwidth.

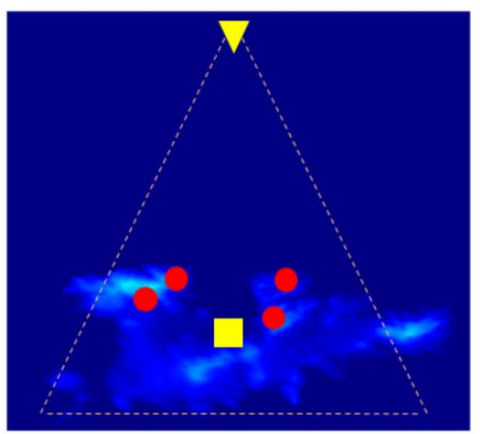

(a)

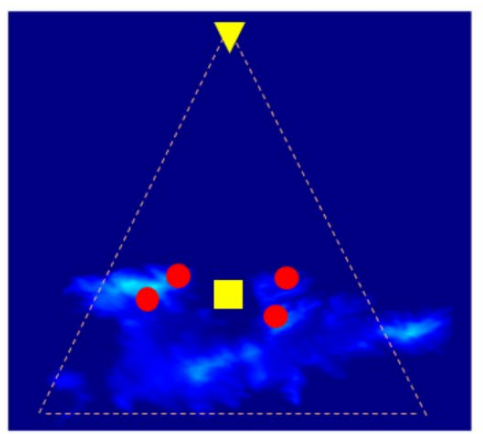

(b)

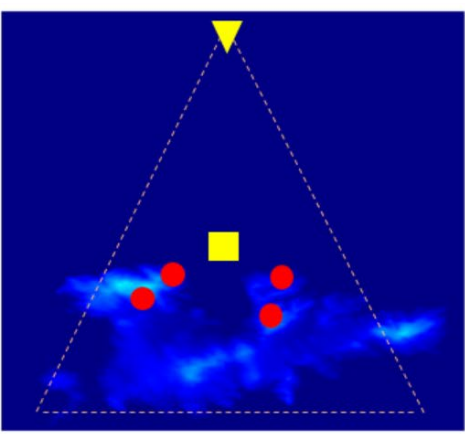

(c)

Figure 6. Placement of the receiver: (a) Behind, (b) Center, (c) Front.

In each experiment, radar parameters were set to simulate the localization geometry as depicted in Figure 1. The first important parameter is the pulse width $(\tau)$ of the radar emitter which was considered to be $1 \mu \mathrm{s}$. The beamwidth $(\theta)$ of the radar emitter which is another important parameter was envisaged to be $10^{\circ}, 15^{\circ}$, and $20^{\circ}$. In addition, the $\mathbf{s}_{0}^{o}$ was considered to measure five different AOA information of radar pulses with an error margin $(\gamma)$ which was defined as $3^{\circ}$. In this case, four probable region of scattering centers were assumed to be obtained $(M=4)$ by excluding the AOA of the direct pulse. Hence, in each experiment, AOA of the multipath pulses were defined according to the position of the scatterers which were randomly selected within the radar cell. However, AOA of the direct pulse was fixed in each scenario $\left(\gamma_{0}=90^{\circ}\right)$.

In the segmentation of the probable region of scattering centers obtained for each scenario, a particular DTED format level is used which corresponds to $22.5 \mathrm{~m} \times$ $22.5 \mathrm{~m}$ segments. It should be noted that the localization method is expected to be more accurate when the small-sized segments are used. The reason of the better accuracy is linked with the lower uncertainty in virtual sensor positions that appears when the small-sized segments are used in the segmentation. Therefore, based on the size of the segments, virtual sensor position noise power is determined as $6 \mathrm{~dB}$ by utilizing basic 
three-sigma rule in statistics to be used in the TDOA technique. Moreover, the distance between the receiver and the radar emitter $\left(r_{0}^{o}\right)$ was envisaged to be $30 \mathrm{~km}, 40 \mathrm{~km}$, and $50 \mathrm{~km}$. Here, the maximum distance was limited to $50 \mathrm{~km}$ in order to avoid signal loss due to earth's curvature. In this case, Furthermore, the possible region of the radar emitter was determined and split up to nine segments $(K=9)$ so that the center of each segment can be assigned as a nominal position of the radar emitter $\left(\mathbf{u}_{k}, k=1, \ldots, K\right)$.

For each scenario, on the other hand, the likelihoods of the scattering centers were estimated by using the presented ray tracing algorithm. From each probable region of virtual sensors, top-five scattering centers that have maximum likelihoods were selected to be used in the localization algorithm. It should be also noted that both the radar emitter and the receiver were assumed to be deployed at naval ships, and their antenna height was assumed to be $60 \mathrm{~m}$. Additionally, according to the position of the scattering centers, heights of the virtual sensors were between $300 \mathrm{~m}$ and $450 \mathrm{~m}$.

Simulations were then performed to evaluate the performance of the localization method. In order to examine the accuracy of the localization algorithm, mean square error (MSE) of the radar emitter location estimate was obtained by using $\operatorname{MSE}(\overline{\overline{\mathbf{u}}})=$ $\sum_{v=1}^{L}\left\|\overline{\overline{\mathbf{u}}}_{v}-\mathbf{u}^{o}\right\|^{2} / L$ where $\overline{\overline{\mathbf{u}}}_{v}$ is the estimated position of $\mathbf{u}^{o}$ in the $v^{\text {th }}$ ensemble run, and $L$ is the number of ensemble runs [24,37]. In the simulations, MSEs of location estimate were obtained at $10^{3}$ ensemble runs. Further, the results were compared with localization MSE of the improved-TSWLS method in order to validate the proposed algorithm. As it is not able to employ the improved-TSWLS method to solve the localization problem in consideration, the localization geometry obtained for each scenario was relaxed. To do this, the center of each probable region of scattering centers was assigned as sensor position to be used in the TSWLS method. 
For the first experiment, the estimation accuracy of the improved-TSWLS and proposed localization method is shown in Figure 7. Based on the results, it can be seen that the proposed method is expected to be inaccurate when the receiver is deployed in the behind of the scatterers as illustrated in Figure 6(a). Even the improved-TSWLS method provides mostly inaccurate results. Nonetheless, one of the important findings is that the localization accuracy of the proposed method tends to increase when the $\theta$ value is increased. Therefore, when the receiver is deployed at the back of the scatterers, the proposed method might provide relatively accurate localization given that the targeted radar emitter has wider beamwidth.
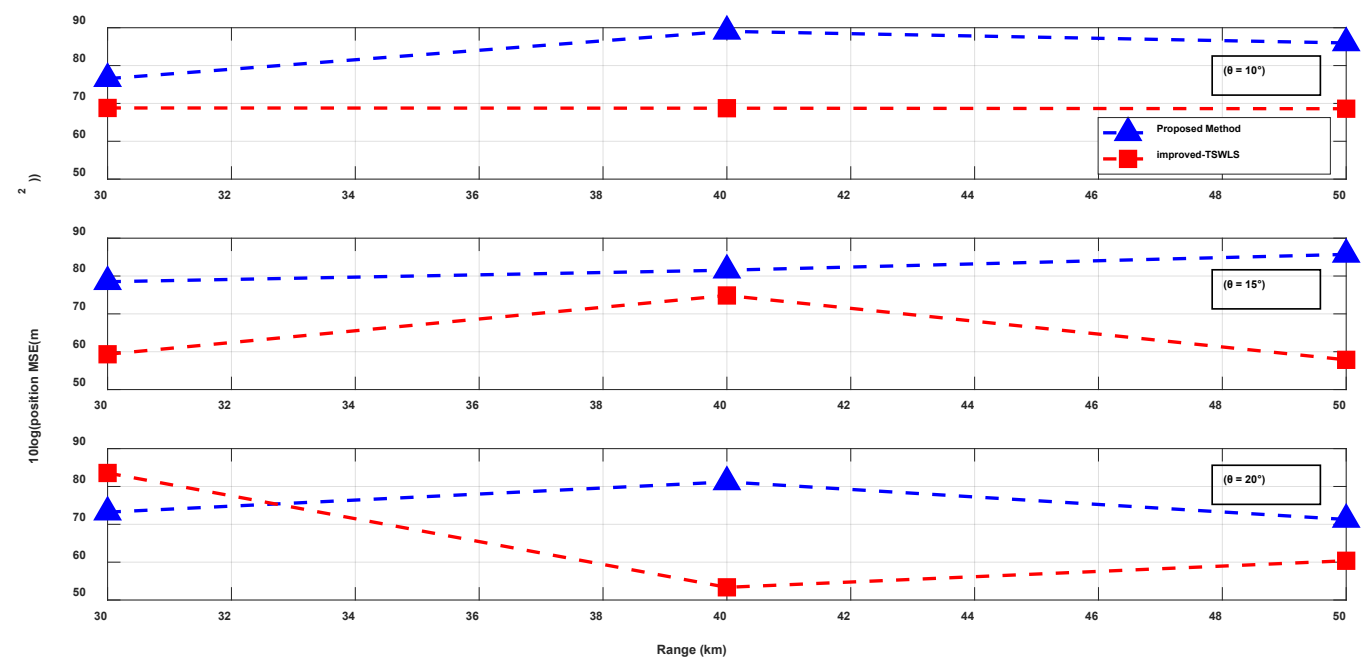

Figure 7. The estimation accuracy of the improved-TSWLS and proposed method when the receiver is in the behind of the scatterers.

Figure 8 shows the accuracy of the improved-TSWLS and proposed method for the second experiment. Inaccurate results obtained by the improved-TSWLS method suggest that a typical TDOA method based on wireless sensor networks could be inefficient for the given localization scenario. However, especially when compared with the previous experiment, the efficiency and the robustness of the proposed method can be clearly observed. For all cases in terms of the range and beamwidth, it provides 
accurate results where the maximum value of estimation error is about $50 \mathrm{~dB}$ which approximately corresponds to $320 \mathrm{~m}$ in distance.

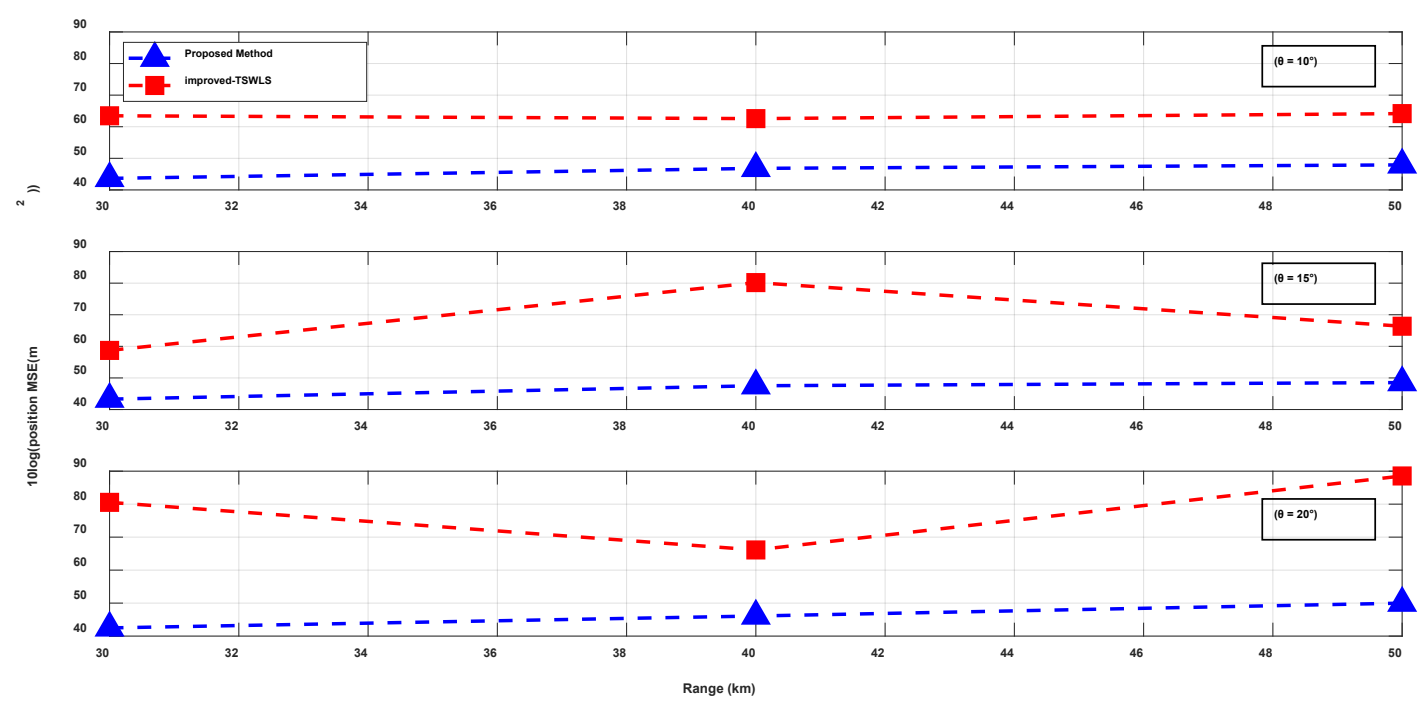

Figure 8 . The estimation accuracy of the improved-TSWLS and proposed method when the receiver is at the center of the scatterers.

In Figure 9, the accuracy of the improved-TSWLS and proposed method for the last experiment is given. It can be observed that the results are inconsistent when $\theta \leq$ $10^{\circ}$. In other words, similar to the results obtained in the previous experiments, the localization accuracy of the proposed method tends to increase when the value of $\theta$ is increased. Thus, the proposed method is expected to be efficient to locate the radar emitter especially at shorter ranges in the case that the receiver is deployed in front of the scatterers. 

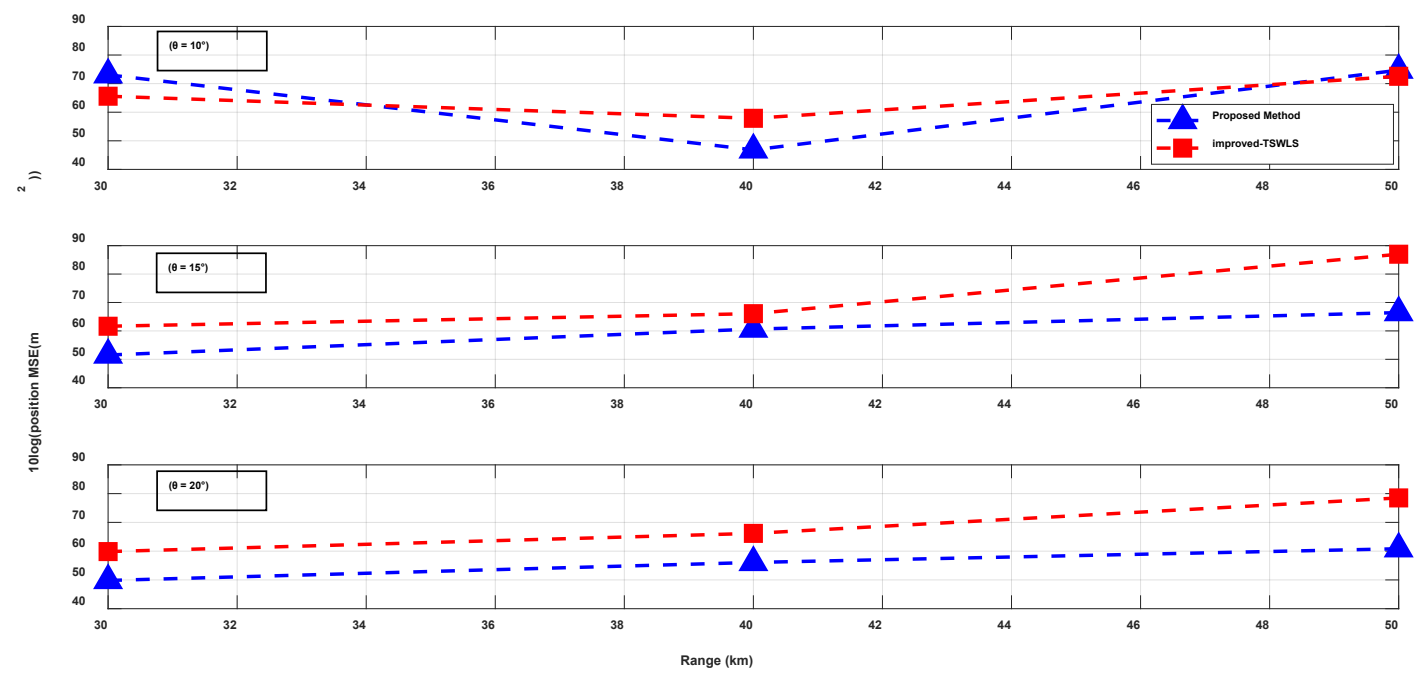

Figure 9. The estimation accuracy of the improved-TSWLS and proposed method when the receiver is in front of the scatterers.

\section{Discussions}

The achieved results from the simulations verify that the localization method is mostly implementable in EW context except some specific localization scenarios. More precisely, although the overall accuracy of the localization method in realistic scenarios is acceptable, the performance of the method is still depended on the position of the virtual sensors. The reason is due to the fact that TDOA-based methods are highly affected the location geometry. Particularly under the poor geometry, the localization accuracy is expected to be degraded. This indeed is the case of large geometric dilution of precision (GDOP) [38]. Generally, GDOP is a metric that is used to evaluate the geometric effect of sensor configurations on the localization accuracy. This means that the localization performance of the system is considered to be worse when the GDOP value is larger. Within this context, the GDOP effect can be easily observed in the simulation results presented in the previous section. Based on the results, the worst accuracy is obtained when the receiver is placed at the center of the virtual sensors. On the other hand, the best accuracy is achieved when the receiver is placed at the center of 
virtual sensors. This can be linked with the virtual sensor layout that yields a lower value of GDOP causing better localization accuracy. This finding is consistent with the previous studies in the literature that examined the GDOP value for various layouts $[39,40]$. Besides, the range between the emitter and the receiver is also important factor that adversely affect the GDOP. Its effects can be clearly observed in the simulation results. Particularly, in each experiment, it is observed that the method provides better accuracy in short-range scenarios.

Furthermore, the localization accuracy of the proposed method could be low when the targeted radar emitter has narrow beamwidth $\left(\theta \leq 10^{\circ}\right)$. This is because, narrower probable region of multipath scattering centers are expected to be obtained when the radar emitter has narrow beamwidth. In this case, virtual sensor positions are expected to be so close to each other leading to poor location geometry, so-called large GDOP. Hence, in order to achieve higher accuracy in the localization systems, it is necessary to acquire good geometric layout between the emitter and sensors. At this point, GDOP can be used as a criterion for selecting proper virtual sensor groups. This can be achieved only if the virtual sensor groups providing minimum GDOP are used in the proposed method. In fact, this constitutes a part of ongoing project, and authors are currently focused on improving the accuracy of the proposed localization method for the case narrower radar beamwidth in real operational scenarios in EW context.

\section{Conclusion}

In this study, the accuracy of an emitter localization method based on multipath exploitation recently proposed by the authors is evaluated in realistic scenarios. Overall, based on the results, it is concluded that the accuracy of the method is acceptable in realistic scenarios and the method is mostly implementable in EW context. Particularly, when the receiver is placed at the center of scatterers, it is expected to achieve better 
accuracy. Besides, the method is also expected to provide better accuracy in short-range scenarios. Moreover, better localization accuracy of the proposed method is expected to be achieved when the targeted radar emitter has wider beamwidth $\left(\theta>10^{\circ}\right)$.

On the other hand, the method is expected to exhibit relatively poor performance when the receiver is placed in the behind of scatterers, and when the targeted radar emitter has narrow beamwidth $\left(\theta \leq 10^{\circ}\right)$. Currently, the authors are extensively seeking new approaches in order to improve the accuracy of the method for such specific localization scenarios.

\section{Acknowledgments}

This work was supported by The Scientific and Technological Research Council of Turkey (TUBITAK) under the grant number 118E157.

\section{References}

[1] Poisel R. Electronic warfare target location methods. Artech House; 2012.

[2] Wang Y, Ho KC. An asymptotically efficient estimator in closed-form for 3-D AOA localization using a sensor network. IEEE Transactions on Wireless Communications. 2015;14:6524-6535.

[3] Chan Y-T, Ho KC. A simple and efficient estimator for hyperbolic location. IEEE Transactions on signal processing. 1994;42:1905-1915.

[4] Ho KC, Xu W. An accurate algebraic solution for moving source location using TDOA and FDOA measurements. IEEE Transactions on Signal Processing. $2004 ; 52: 2453-2463$.

[5] Wang Y, Ho KC. Unified near-field and far-field localization for AOA and hybrid AOA-TDOA positionings. IEEE Transactions on Wireless Communications. $2017 ; 17: 1242-1254$. 
[6] Rigling BD. Urban RF multipath mitigation. IET Radar, Sonar \& Navigation. $2008 ; 2: 419-425$.

[7] Waldmann B, Goetz A, Weigel R. An ultra wideband positioning system enhanced by a short multipath mitigation technique. 2009 IEEE MTT-S International Microwave Workshop on Wireless Sensing, Local Positioning, and RFID. IEEE; 2009. p. 1-4.

[8] Yu J, Krolik J. MIMO multipath clutter mitigation for GMTI automotive radar in urban environments. 2012;

[9] Guo S, Cui G, Kong L, et al. An imaging dictionary based multipath suppression algorithm for through-wall radar imaging. IEEE Transactions on Aerospace and Electronic Systems. 2017;54:269-283.

[10] Guo S, Yang X, Cui G, et al. Multipath ghost suppression for through-the-wall imaging radar via array rotating. IEEE Geoscience and Remote Sensing Letters. $2018 ; 15: 868-872$.

[11] Copa EIP, Aziz K, Rykunov M, et al. Radar Fusion for Multipath Mitigation in Indoor Environments. 2020 IEEE Radar Conference (RadarConf20). IEEE; 2020. p. 15.

[12] Tan CKS and SY. Localization of Omni-Directional Mobile Device in Multipath Environments. Progress In Electromagnetics Research. 2008;85:323-348.

[13] Shen Y, Win MZ. On the Use of Multipath Geometry for Wideband Cooperative Localization. GLOBECOM 2009 - 2009 IEEE Global Telecommunications Conference. 2009. p. 1-6.

[14] Lui KW, So HC. Range-based source localisation with pure reflector in presence of multipath propagation. Electronics Letters. 2010;46:957-958. 
[15] Agate CS, Varble M, Ezal KO. Ground-Based Emitter Location in the Presence of Multipath. 2019 IEEE Aerospace Conference. 2019. p. 1-8.

[16] Meissner P, Witrisal K. Multipath-assisted single-anchor indoor localization in an office environment. 2012 19th International Conference on Systems, Signals and Image Processing (IWSSIP). 2012. p. 22-25.

[17] Setlur P, Smith GE, Ahmad F, et al. Target Localization with a Single Sensor via Multipath Exploitation. IEEE Transactions on Aerospace and Electronic Systems. 2012;48:1996-2014.

[18] O'connor A, Setlur P, Devroye N. Single-sensor RF emitter localization based on multipath exploitation. IEEE Transactions on Aerospace and Electronic Systems. $2015 ; 51: 1635-1651$.

[19] Muqaibel AH, Amin MG, Ahmad F. Target Localization with a Single Antenna via Directional Multipath Exploitation [Internet]. International Journal of Antennas and Propagation. Hindawi; 2015 [cited 2021 Mar 1]. p. e510720. Available from: https://www.hindawi.com/journals/ijap/2015/510720/.

[20] Giacometti R, Baussard A, Jahan D, et al. Localization of radar emitters from a single sensor using multipath and TDOA-AOA measurements in a naval context. 2016 24th European Signal Processing Conference (EUSIPCO). 2016. p. 692-696.

[21] Nikoo MS, Behnia F. Single-site source localisation using scattering data. IET Radar, Sonar \&amp; Navigation. 2017;12:250-259.

[22] Sousa MN de, Thoma RS. Single Sensor RF Emitter Location Using Ray Tracing Multipath Exploitation. 2018 15th International Symposium on Wireless Communication Systems (ISWCS). 2018. p. 1-6.

[23] Dalveren Y, Kara A. Multipath Exploitation in Emitter Localization for Irregular Terrains. RADIOENGINEERING. 2019;27:473-482. 
[24] Liu Y, Guo F, Yang L, et al. An improved algebraic solution for TDOA localization with sensor position errors. IEEE Communications Letters. 2015;19:22182221.

[25] Polka LA, Balanis CA, Polycarpou AC. High-frequency methods for multiple diffraction modeling: application and comparison. Journal of Electromagnetic Waves and Applications. 1994;8:1223-1246.

[26] Kam K-Y, Chew L-Y, Li L-W. Multipath Propagation of Radio Waves in 3Dimensional Terrains. Journal of Electromagnetic Waves and Applications. $2001 ; 15: 411-431$.

[27] Tabakcioglu MB, Kara A. Comparison of improved slope uniform theory of diffraction with some geometrical optic and physical optic methods for multiple building diffractions. Electromagnetics. 2009;29:303-320.

[28] Tabakcioglu MB, Kara A. Improvements on slope diffraction for multiple wedges. Electromagnetics. 2010;30:285-296.

[29] Tabakcioglu MB. S-UTD-CH model in multiple diffractions. International Journal of Electronics. 2016;103:765-774.

[30] Azpilicueta L, Aguirre E, López-Iturri P, et al. An accurate UTD extension to a ray-launching algorithm for the analysis of complex indoor radio environments. Journal of Electromagnetic Waves and Applications. 2016;30:43-60.

[31] Rizk K, Valenzuela R, Chizhik D, et al. Application of the slope diffraction method for urban microwave propagation prediction. VTC '98 48th IEEE Vehicular Technology Conference Pathway to Global Wireless Revolution (Cat No98CH36151). 1998. p. 1150-1155 vol.2. 
[32] Kouyoumjian RG, Pathak PH. A uniform geometrical theory of diffraction for an edge in a perfectly conducting surface. Proceedings of the IEEE. 1974;62:14481461.

[33] Tzaras C, Saunders SR. An improved heuristic UTD solution for multiple-edge transition zone diffraction. IEEE Transactions on Antennas and Propagation. 2001;49:1678-1682.

[34] McNamara DA, Malherbe JAG, Pistorius CW. Introduction to the uniform geometrical theory of diffraction. Artech House; 1990.

[35] Dalveren Y, Kara A. Comparative analysis of TDOA-based localization methods in the presence of sensor position errors. 2017 4th International Conference on Control, Decision and Information Technologies (CoDIT). 2017. p. 0556-0560.

[36] Trivedi KS. Probability and statistics with reliability, queuing, and computer science applications. Wiley Online Library; 2002.

[37] Ho KC, Lu X, Kovavisaruch L. Source localization using TDOA and FDOA measurements in the presence of receiver location errors: Analysis and solution. IEEE Transactions on Signal Processing. 2007;55:684-696.

[38] Sharp I, Yu K, Guo YJ. GDOP analysis for positioning system design. IEEE Transactions on Vehicular Technology. 2009;58:3371-3382.

[39] Lei Y-P, Gong F-X, Ma Y-Q. Optimal distribution for four-station TDOA location system. 2010 3rd International Conference on Biomedical Engineering and Informatics. IEEE; 2010. p. 2858-2862.

[40] Levanon N. Lowest GDOP in 2-D scenarios. IEE Proceedings-radar, sonar and navigation. 2000;147:149-155. 